\title{
The Triaxial Rotation Vibration Model in the Xe-Ba Region
}

\author{
U. Meyer円, Amand Faessler, S.B. Khadkikar \\ Institute for Theoretical Physics, University of Tübingen \\ Auf der Morgenstelle 14, D-72076 Tübingen, Germany
}

\begin{abstract}
Collective quadrupole degrees of freedom give rise to vibrations and rotations in nuclei. The axial Rotation Vibration Model (RVM) is here extended to describe also triaxial equilibrium shapes with $\beta$ and $\gamma$ vibrations allowing for the interaction between vibrations and rotations. This Triaxial Rotation Vibration Model (TRVM) is applied to $\mathrm{Ba}$ and Xe isotopes with $\mathrm{A} \approx 120$ to 130 . This area has recently been pointed out to be a region for the $\mathrm{O}(6)$ limit of the Interacting Boson Approximation (IBA). The present work shows that the TRVM can equally well describe these nuclei concerning their excitation energies and E2 branching ratios.
\end{abstract}

PACS numbers: 21.60 Ev, 23.20.-g, 23.20.Js, 27.60.+j

Keywords: Collective nuclear models, triaxiallity, Rotation Vibration Model, Xe-Ba nuclei, energy levels, E2 branching ratios.

${ }^{1}$ Supported by the DFG Graduiertenkolleg MU 705/3 


\section{Introduction}

The collective quadrupole degrees in nuclei yield rotational and vibrational excitations. They are described by the Bohr-Mottelson Hamiltonian [1]. The wavefunctions for rotations and $\beta$ and $\gamma$ vibrations in strongly deformed axially symmetric nuclei have been greatly improved in the Rotation Vibration Model (RVM) by Faessler and Greiner [2, 3, 4, 5] taking the interaction between the rotations and vibrations into account with an axially symmetric equilibrium deformation.

Recently, the $\mathrm{Ba}$ and Xe region with mass numbers $\mathrm{A} \approx 120$ to 130 has been studied experimentally [6, 9-15] and interpreted by several models: They compare different approaches of the Interacting Boson Approximation (IBA) in the $\mathrm{U}(5)$ and the $\mathrm{O}(6)$ limits (with different E2 operators) [7] and the axial Rotation Vibration Model (RVM) and the Asymmetric Rotor Model (ARM) [8]. They exclude the ARM [9, 11] because it cannot describe a $\mathrm{K}=0$ band built on $\gamma$ vibrations [11] and because of the wrong staggering of the $2^{+}, 3^{+}, 4^{+}, 5^{+}, \ldots$ excitation energies in the $\mathrm{K}=2$ band. The argument with the $\mathrm{K}=0$ band built on $\gamma$ vibrations is trivial: The ARM does not contain $\gamma$ vibrations and thus cannot describe it. Because the staggering in the $(K=2)$ quasi $\gamma$ band is not described correctly in the ARM, but given correctly in the RVM, the staggering seems to be due to rotation vibration interaction. Although the RVM [2, 3, 4, 5] does quite well in describing the data, the Cologne group concludes [11] that the $\mathrm{O}(6)$ IBA with the additional parameter $\chi$ in the $\mathrm{E} 2$ transition operator does in average agree better with the data. Here, we want to show that this is connected with the restriction of the RVM [2, 3, 4, 5] to axial symmetry while IBA allows also for triaxiallity $(\mathrm{O}(6)=\gamma$ instable limit). We present here an extension of the axial RVM to triaxiallity (Triaxial Rotation Vibration Model = TRVM). This model has the same number of parameters as the IBA used [9, 11]. We obtain an equally good agreement with the data as in IBA.

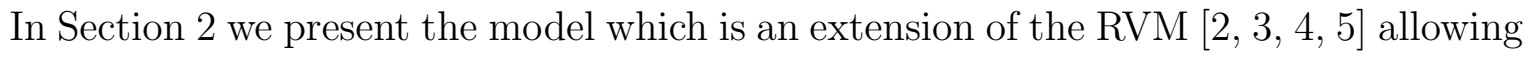
for a triaxial equilibrium deformation (TRVM). In Section 3 we compare the excitation energies and the branching ratios in the best measured $\mathrm{Xe}$ and $\mathrm{Ba}$ isotopes. Section 4 summarizes the main results.

\section{The Triaxial Rotation Vibration Model}

In the Rotation Vibration Model [2, 3, 4, 5] one characterizes as in the Bohr-Mottelson Model [1] the surface of the nucleus by quadrupole deformations:

$$
R(\theta, \phi)=R_{0}\left(1+\sum_{\mu} \alpha_{2 \mu} Y_{2 \mu}(\theta, \phi)\right)
$$

The deformation parameters $\alpha_{2 \mu}$ are considered as dynamical variables and depend classically on time. Their behaviour is governed by the Hamiltonian:

$$
H=\frac{1}{2} B \sum_{\mu} \dot{\alpha}_{2 \mu}^{\dagger} \dot{\alpha}_{2 \mu}+V\left(\alpha_{2 \mu}\right)
$$

The information about the equilibrium shape of the nucleus is contained in the potential energy $V\left(\alpha_{2 \mu}\right)$. The five quadrupole degrees of freedom $\alpha_{2 \mu}$ can be replaced by the three Euler angles $(\phi, \theta, \psi)$ for rotations and two deformation parameters

$$
\alpha_{2 \mu}=\mathcal{D}_{\mu 0}^{2}(\phi, \theta, \psi)\left(\beta_{0}+a_{0}^{\prime}(t)\right)+\left(\mathcal{D}_{\mu 2}^{2}+\mathcal{D}_{\mu-2}^{2}\right)\left(a_{2}+a_{2}^{\prime}(t)\right)
$$


Here $\beta_{0}$ and $a_{2}$ give the equilibrium shape of the nucleus while $a_{0}^{\prime}(t)$ and $a_{2}^{\prime}(t)$ describe vibrations around this shape. They are connected with the Bohr-Mottelson parameters through:

$$
\begin{aligned}
\beta_{0}+a_{0}^{\prime}(t) & =\beta \cos (\gamma) \\
a_{2}+a_{2}^{\prime}(t) & =\frac{1}{\sqrt{2}} \beta \sin (\gamma)
\end{aligned}
$$

In deriving the Hamiltonian for the TRVM we follow very closely the derivation for the axial RVM in Ref. 41. With the transformation (3) the Hamiltonian (2) can be written (see in Ref. [1] eqs. (3) and (4)):

$$
H=T+V=T_{\text {rot }}+T_{v i b}+T_{\text {rotvib }}+V_{a_{0} a_{2}}\left(a_{0}^{\prime}, a_{2}^{\prime}\right)
$$

The different terms are obtained by the straight forward transformation (3) and the expansion in powers of $a_{0}^{\prime} / b_{0}$ and $a_{2}^{\prime} / a_{2}$ up to second order. This assumes that the $\beta$ and $\gamma$ deformation is large compared to the vibrational amplitudes. This approach therefore does not allow to describe spherical or nearly spherical nuclei. The several terms in the TRVM Hamiltonian are given by:

$$
\begin{aligned}
T_{\text {rot }}= & \frac{\hat{\mathbf{I}}^{2}-\hat{I}_{3}^{2}}{2 I_{0}}+\frac{\hat{I}_{3}^{2}}{16 B a_{2}^{2}} \\
T_{\text {vib }}= & -\frac{\hbar^{2}}{2 B}\left(\frac{\partial^{2}}{\partial a_{0}^{\prime 2}}+\frac{1}{2} \frac{\partial^{2}}{\partial a_{2}^{\prime 2}}\right) \\
T_{\text {rotvib }}= & \frac{\hat{\mathbf{I}}^{2}-\hat{I}_{3}^{2}}{2 I_{0}} f_{0}\left(\beta_{0}, a_{2}, a_{0}^{\prime}, a_{2}^{\prime}\right) \\
& +\frac{\hat{I}_{+}^{2}+\hat{I}_{-}^{2}}{2 I_{0}} f_{1}\left(\beta_{0}, a_{2}, a_{0}^{\prime}, a_{2}^{\prime}\right) \\
& +\frac{\hat{I}_{3}^{2}}{16 B a_{2}^{2}} f_{2}\left(a_{2}, a_{2}^{\prime}\right)+2 \epsilon \frac{a_{0}^{\prime}}{\beta_{0}}
\end{aligned}
$$

The functions $f_{0}, f_{1}$, and $f_{2}$ are obtained by the expansion mentioned above:

$$
\begin{aligned}
& f_{0}=-2 \frac{a_{0}^{\prime}}{\beta_{0}}+3 \frac{a_{0}^{\prime^{2}}}{\beta_{0}^{2}}+\frac{2}{\beta_{0}^{2}}\left(a_{2}^{2}+2 a_{2} a_{2}^{\prime}+a_{2}^{\prime^{2}}\right) \\
& f_{1}=\frac{1}{3} \sqrt{6} \frac{1}{\beta_{0}}\left(a_{2}+a_{2}^{\prime}\right)-\sqrt{6} \frac{1}{\beta_{0}^{2}} a_{0}^{\prime}\left(a_{2}+a_{2}^{\prime}\right) \\
& f_{2}=-2 \frac{a_{2}^{\prime}}{a_{2}}+3 \frac{a_{2}^{\prime 2}}{a_{2}^{2}}
\end{aligned}
$$

For the potential energy we assume a harmonic oscillator potential around the equilibrium shape:

$$
V\left(a_{0}^{\prime}, a_{2}^{\prime}\right)=\frac{1}{2} C_{0} a_{0}^{\prime^{2}}+C_{2} a_{2}^{\prime^{2}}
$$

For the diagonalization of the Hamiltonian we chose a basis of eigenstates of the Hamiltonian $H_{0}=T_{\text {rot }}+T_{v i b}+V$. The eigenstates of the unperturbed Hamiltonian $H_{0}$ are easily 
obtained and labelled by the total angular momentum $I$, the projection on the intrinsic 3 -axis $K$ and the number of phonons for the $\beta$ - $\left(n_{0}\right)$ and $\gamma$ vibrations $\left(n_{2}\right)$ :

$$
\left|I K, n_{2} n_{0}\right\rangle=\left(\frac{2 I+1}{16 \pi^{2}} \frac{1}{1+\delta_{K 0}}\right)^{\frac{1}{2}}\left(\mathcal{D}_{M K}^{I}+(-)^{I} \mathcal{D}_{M-K}^{I}\right) \sqrt{\frac{1}{n_{0} !}}\left(\hat{\beta}_{0}^{\dagger}\right)^{n_{0}}|0\rangle \sqrt{\frac{1}{n_{2} !}}\left(\hat{\beta}_{2}^{\dagger}\right)^{n_{2}}|0\rangle
$$

Due to the rotation vibration part of the Hamiltonian $T_{\text {rotvib }}$ several eigenstates of $H_{0}$ are mixed. The unperturbed energies as eigenvalues of $H_{0}$ may easily be obtained. They are given by:

$$
\begin{aligned}
E_{n_{2} n_{0}}^{I K} & =\left(I(I+1)-K^{2}\right) \frac{\hbar^{2}}{2 I_{0}}+\frac{K^{2}}{16 B a_{2}^{2}}+\left(n_{0}+\frac{1}{2}\right) E_{\beta}+\left(n_{2}+\frac{1}{2}\right) E_{\gamma} \\
\text { with } I & =\left\{\begin{array}{l}
0,2,4,6, \ldots \ldots \ldots \ldots \\
K, K+1, K+2, \ldots
\end{array} \text { for } \mathrm{K}=0\right. \\
E_{\beta}=\hbar \sqrt{\frac{C_{0}}{B}} & ; E_{\gamma}=\hbar \sqrt{\frac{C_{2}}{B}} \\
\epsilon & =\frac{\hbar^{2}}{I_{0}} \\
I_{0} & =3 B \beta_{0}^{2}
\end{aligned}
$$

The operators $\hat{\beta}^{\dagger}$ are creation operators for harmonic oscillations. We diagonalize the Hamiltonian (5), (6), (7) in the complete basis (8). For numerical reasons the Hilbert space has to be truncated. We chose the 31 lowest basis states with $K \leq 6$ and up to two phonons $\left(n_{2}+n_{0} \leq 2\right)$. In notation (8) the most important states $\left|I K, n_{2} n_{0}\right\rangle$ are:

$$
\begin{array}{lll}
|I 0,00\rangle & \ldots \ldots & \text { ground state band } \\
|I 2,00\rangle & \ldots \ldots & \mathrm{K}=2 \text { quasi } \gamma \text { band } \\
|I 0,10\rangle & \ldots \ldots & \text { one-phonon } \gamma \text { band } \\
|I 0,01\rangle & \ldots \ldots & \text { one-phonon } \beta \text { band } \\
|I 4,00\rangle & \ldots \ldots & \mathrm{K}=4 \text { quasi } \gamma \text { band }
\end{array}
$$

The TRVM has as the IBA four independent parameters. The vibration energies $E_{\beta}$ and $E_{\gamma}$, the inverse moment of inertia $\epsilon=1 / I_{0}$ and the triaxial equilibrium deformation $a_{2}$.

The Hilbert space is too limited to describe the full variation of the moment of inertia. As in the competing IBA [13] we make for the energies the Lipas ansatz

$$
E=E_{0} /\left(1+\alpha_{L} E_{0}\right)
$$

$E_{0}$ is the excitation energy obtained after diagonalization. The Lipas parameter $\alpha_{L}$ is quite small $\left(\approx 10^{-4}\left[\mathrm{keV}^{-1}\right]\right)$. It describes a variable moment of inertia. We would like to note that the number of parameters including the Lipas parameter is the same in TRVM and in IBA (including the effective boson charge in IBA). In the $\mathrm{O}(6)$ limit the branching ratios of IBA depend only on the parameter $\chi$. But similarly most of the branching ratios in TRVM depend essentially only on the effective triaxiallity $a_{2}+a_{2}^{\prime}$. In addition, one could omit practically the $\beta$ band and therefore the parameter $E_{\beta}$ in TRVM, since the results for the branching ratios do almost not depend on the $\beta$ band and the agreement would be equally good by omitting this band. But on the other side, this means also that our prediction for the $\beta$ bandhead is not reliable. Both models therefore effectively have the same number of parameters. 


\section{Comparison with the Data}

\subsection{Energy spectra}

In Figure 1 we display our results for the energy spectrum of ${ }^{130} \mathrm{Ba}$. The free parameters which give an overall best fit of the experimental data from Ref. 113 are listed in Table 1. For comparison, in Figure 1 furthermore the calculated IBA values and the experimental results are shown. For the low spin states in the ground state band both theoretical results are in nice agreement with experiment. As a result of the applied Lipas fitting procedure lower spin states as the $4^{+}, 6^{+}$and also the $8^{+}$state lie slightly higher while the $10^{+}$-state is little below the experimental values. This is due to the stronger effect of the Lipas procedure on higher spin states.

For the excited $K=2$ band (quasi $\gamma$ band) we obtain in TRVM the experimentally observed staggering of even-odd angular momentum states. A pure triaxial rotor model without vibrations but with a $\gamma$ deformation of the nuclear surface does not exhibit this feature. The staggering thus seems to be the result of the rotation vibration coupling. We would like to note that the staggering increases with excitation energies in TRVM. In contrast, in IBA the staggering seems to be constant with growing excitation energy. Both models agree qualitatively with the data in the $\gamma$ band but not in detail.

The $K=0$ band is interpreted in the TRVM as the $\gamma$ one-phonon band. The bandhead $\left(0^{+}\right)$at $1179 \mathrm{keV}$ is fitted through the vibration energy $E_{\gamma}$. For the TRVM the $0^{+}-2^{+}$ splitting is nicely reproduced and in better agreement with experiment than IBA. The $\beta$ vibrational bandhead is at around $1150 \mathrm{keV}$. Up to now, the levels of this band have not been identified in the experimental analysis which might be difficult because the two $0^{+}$ energies are very close. Since our values for the experimentally observed energy levels are almost not affected by the $\beta$ band, we do not display it in Figure 1.

In Figure 2 we show our results for the ${ }^{126}$ Xe spectrum. There a different set of parameters must be used in order to fit the experimental data best. This set is displayed in Table 2. The agreement with the experimental level spacings is in the two theoretical models about the same. This holds for the ground state band as well as for the quasi $\gamma$ band. In TRVM the staggering in low lying states is rather low. The free parameters $a_{2} / \beta_{0}$ and $E_{\beta}$ have to be increased relative to these in ${ }^{130} \mathrm{Ba}$ to get the best agreement. The excited $0^{+}$band is in good agreement with experiment and the level spacings obtained in TRVM are in better agreement with the experiment than the IBA calculations.

Table 2

Figure 2

\subsection{E2 Branching Ratios}

The electric quadrupole operator can again be obtained from the one in Ref. [4] by replacing $a_{2}^{\prime} \rightarrow a_{2}+a_{2}^{\prime}$. In collective intrinsic coordinates, it is given by:

$$
\begin{aligned}
m(E 2, \mu)= & \frac{3 Z}{4 \pi} R_{0}^{2}\left[\mathcal{D}_{\mu 0}^{2}\left(\beta_{0}\left(1+\frac{2}{7}\left(\frac{5}{\pi}\right)^{\frac{1}{2}} \beta_{0}\right)\right)+\mathcal{D}_{\mu 0}^{2} a_{0}^{\prime}\left(1+\frac{4}{7}\left(\frac{5}{\pi}\right)^{\frac{1}{2}} \beta_{0}\right)+\right. \\
& \mathcal{D}_{\mu 0}^{2} \frac{2}{7}\left(\frac{5}{\pi}\right)^{\frac{1}{2}}\left(a_{0} \prime^{2}-2\left(a_{2}+a_{2}^{\prime}\right)^{2}\right)+
\end{aligned}
$$

Table 1

Figure1 


$$
\left.\left(\mathcal{D}_{\mu 2}^{2}+\mathcal{D}_{\mu-2}^{2}\right)\left(\left(1-\frac{4}{7}\left(\frac{5}{\pi}\right)^{\frac{1}{2}} \beta_{0}\right)\left(a_{2}+a_{2}^{\prime}\right)-\frac{4}{7}\left(\frac{5}{\pi}\right)^{\frac{1}{2}} a_{0}^{\prime}\left(a_{2}+a_{2}^{\prime}\right)\right)\right]
$$

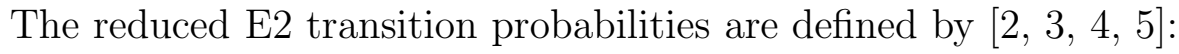

$$
B\left(E 2 ; I_{i} \rightarrow I_{f}\right)=\frac{2 I_{f}+1}{2 I_{i}+1}\left|\left\langle I_{i}|| m(E 2)|| I_{f}\right\rangle\right|^{2}
$$

We have calculated the measured $\mathrm{B}(\mathrm{E} 2)$ ratios to be able to compare them with the experimental results for both ${ }^{130} \mathrm{Ba}$ and ${ }^{126} \mathrm{Xe}$. In Table 3 the results for ${ }^{130} \mathrm{Ba}$ are given. We see a fair agreement between the results of TRVM and the experiment as well as those of IBA except for the transition from the initial $4_{3}^{+}$state. Here our result is about two orders of magnitude larger while the IBA result is two orders of magnitude lower than the experimental ratio $\mathrm{B}\left(\mathrm{E} 2,4_{3}^{+} \rightarrow 2_{2}^{+}\right) / \mathrm{B}\left(\mathrm{E} 2,4_{3}^{+} \rightarrow 4_{2}^{+}\right)$. A similar discrepancy exists also for the transition $\left(4_{3}^{+} \rightarrow 2_{1}^{+}\right)$as can be seen from the last entry in Table 3. Any B(M1)

contribution to the $4_{3}^{+} \rightarrow 4_{2}^{+}$transition cannot cure this disagreement.

Table 3

Table 4 shows the ratios of certain $\mathrm{B}(\mathrm{E} 2)$ transitions in ${ }^{126} \mathrm{Xe}$. Here the agreement is much better between the models and the experiment; and both Interacting Boson Approximation and the Triaxial Rotation Vibration Model agree nicely with the data. This might be because only pure quadrupole transitions are used in Xe to define the ratios, while the uncertainty in the E2/M1 ratio in the normalising states is ignored in the case of $\mathrm{Ba}$ and transitions are assumed to be pure $\mathrm{E} 2$ transitions.

Table 4

\section{Conclusions}

The Triaxial Rotation Vibration Model (TRVM) is successfully applied to Ba and Xe nuclei to obtain low lying energy levels. These results compare well with those obtained in the $\mathrm{O}(6)$-limit of IBA and with the experimental values both for the spectra and for relative E2 transition rates. The bandhead of the $\beta$ vibrational band are not seen in experiment so far. Unfortunately, we are not able to specify very exactly the energy region for the bandhead or other levels of the $\beta$ band since our calculated values are not very sensitive to the parameter $E_{\beta}$.

Acknowledgment: We would like to thank Dr. Ingo Wiedenhöver and Prof. Peter von Brentano for providing us the IBA results and for fruitful discussions.

\section{References}

[1] A. Bohr, B. Mottelson, Dan. Mat. Fys. Medd. 27 (1953) No.16

[2] A. Faessler, W. Greiner, Z. Phys. 168 (1962) 425

[3] A. Faessler, W. Greiner, Z. Phys. 170 (1962) 105 and 177 (1964) 190

[4] A. Faessler, W. Greiner, R.K. Sheline, Nucl. Phys. 70 (1965) 33 
[5] A. Faessler, W. Greiner, R.K. Sheline, Phys. Rev. 135 (1964) B591 and Nucl. Phys. 62 (1965) 241

[6] R.F. Casten, P. von Brentano, Phys. Lett. B152 (1985) 22

[7] F. Iachello, A. Arima, "The Interacting Boson Model", Cambridge Univ. Press, 1987

[8] A.S. Dawydow, V.S. Rostovsky, Nucl. Phys. 12 (1959) 58, A.S. Dawydow, Nucl. Phys. 24 (1961) 682 and A.S. Dawydow, A.A. Chaban Nucl. Phys. 20 (1960) 499

[9] W. Lieberz, A. Dewald, W. Frank, A. Gelberg, W. Krips, D. Lieberz, R. Wirowski and P. von Brentano, Phys. Lett. B240 (1990) 38

[10] A. Dewald, P. Sala, R. Wrzal, G. Böhm, D. Lieberz, G. Siems, W. Wirowski, K.O. Zell, A. Gelberg, P. von Brentano, P. Nolan, A.J. Kirwan, P.J. Bishop, R. Julin, A. Lampinen, J. Hattula, Nucl. Phys. A545 (1992) 822

[11] F. Seiffert, W. Lieberz, A. Dewald, S. Freund, A. Gelberg, A. Granderath, D. Lieberz, R. Wirowski and P. von Brentano, Nucl. Phys. A554 (1993) 287

[12] G. Siems, U. Neuneyer, I. Wiedenhöver, S. Albers, M. Eschenauer, R. Wirowski, A. Gelberg, P. von Brentano, Phys. Lett. B320 (1994) 1

[13] K. Kirch, G. Siems, M. Eschenauer, A. Gelberg, R. Kühn, A. Mertens, U. Neuneyer, O. Vogel, I. Wiedenhöver, P. von Brentano, T. Otsuka, Nucl. Phys. A587 (1995) 211

[14] R. Kühn, K. Kirch, I. Wiedenhöver, O. Vogel, M. Wilhelm. U. Neuneyer, M. Luig, A. Gelberg, P. von Brentano, Nucl. Phys. A597 (1996) 85

[15] A. Dewald, D. Weil, R. Krücken, R. Kühn, R. Peusquens, H. Tiesler, O. Vogel, K.O. Zell, P. von Brentano, D. Bazzacco, C. Rossi-Alvarez, P. Pavan, D. DeAcuña, G. De Angelis, M. De Poli, "A Dynamic Shape Effect in ${ }^{126}$ Ba at Low-Spin", preprint 1996 
${ }^{130} \mathrm{Ba}$

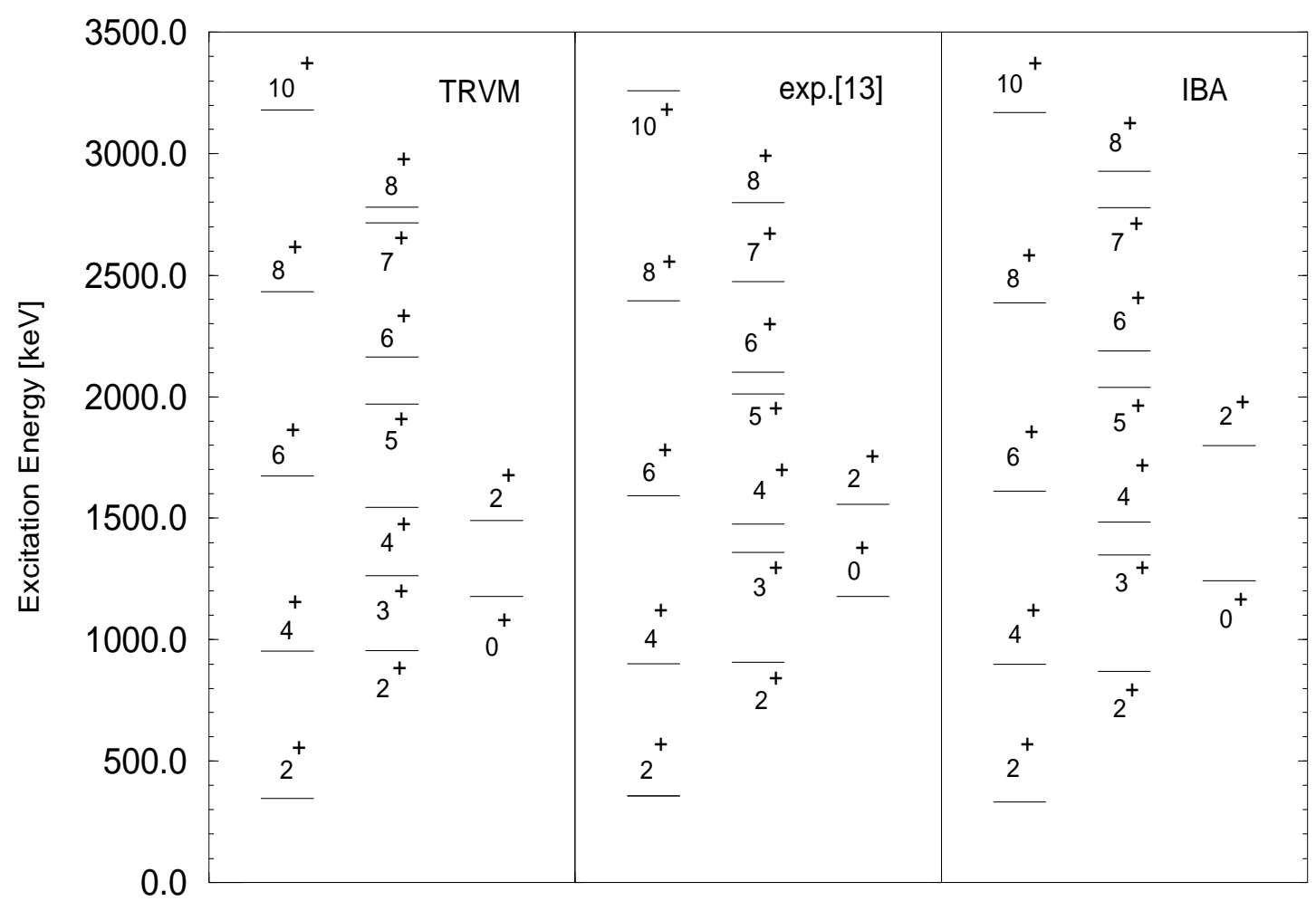

Fig.1: Ground state, quasi $\gamma$ and $K=0$ band for ${ }^{130} \mathrm{Ba}$ in $\mathrm{keV}$ for the IBA calculation and the Triaxial Rotation Vibration Model (TRVM) compared with experimental values from Ref.[13]. 
${ }^{126} \mathrm{Xe}$

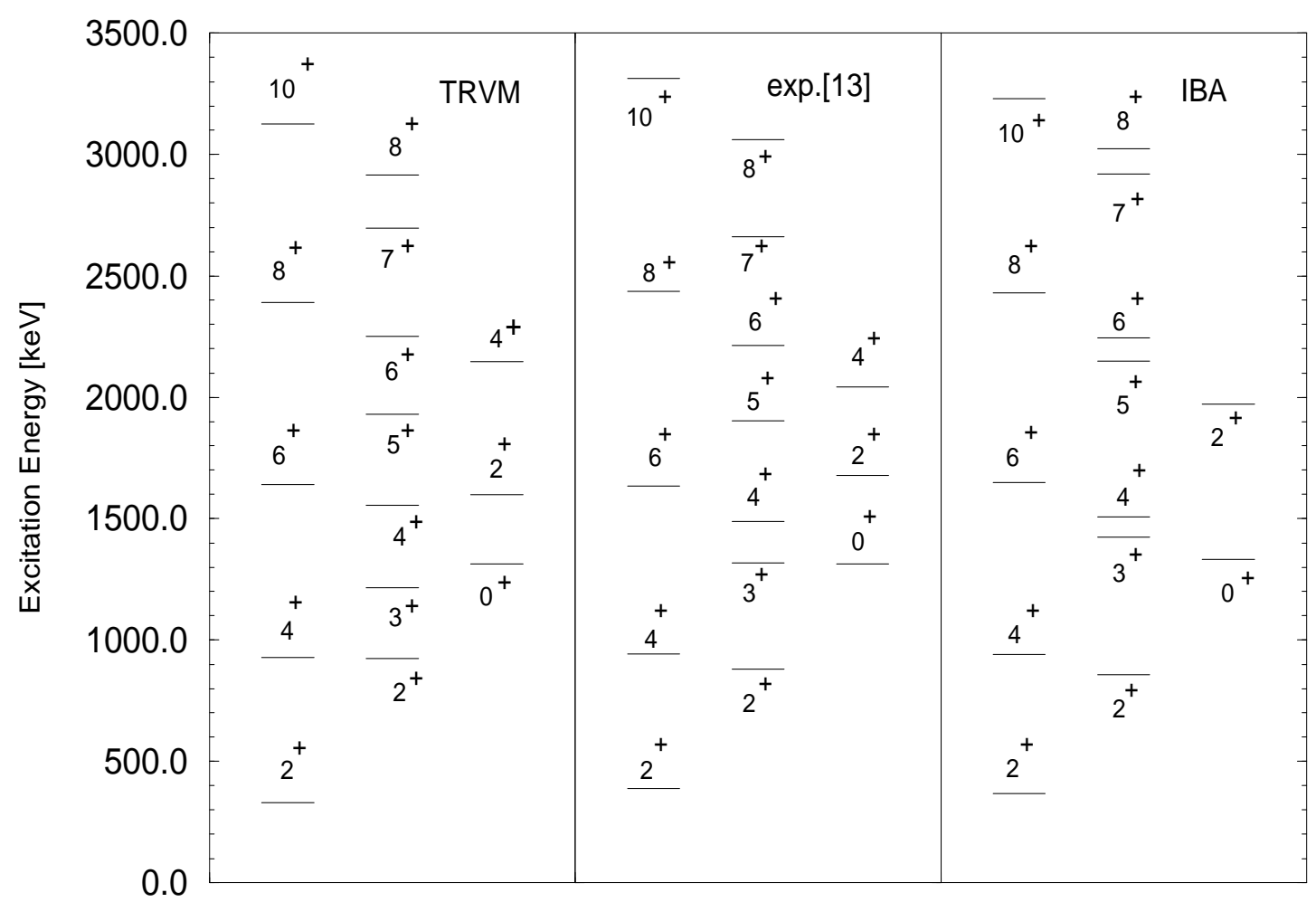

Fig.2: Ground state, quasi $\gamma$ and $K=0$ band for ${ }^{126} \mathrm{Xe}$ in $\mathrm{keV}$ for the IBA calculation and the Triaxial Rotation Vibration Model (TRVM) compared with experimental values from Ref.[13]. 


\begin{tabular}{|c|c|c|c|c|}
\hline$E_{\beta}[\mathrm{keV}]$ & $E_{\gamma}[\mathrm{keV}]$ & $\epsilon[\mathrm{keV}]$ & $a_{2} / \beta_{0}$ & $\alpha_{L}\left[\mathrm{keV}^{-1}\right]$ \\
\hline 1168 & 1179 & 80.5 & 0.329 & $10^{-4}$ \\
\hline
\end{tabular}

Table 1: Parameters of the TRVM in the present calculations for ${ }^{130} \mathrm{Ba}$. $E_{\beta}$ and $E_{\gamma}$ are the vibration energies, $\epsilon$ is the inverse moment of inertia and $a_{2} / \beta_{0}$ the relative ratio of the static $\gamma$ and $\beta$ deformations. $\alpha_{L}$ is the Lipas parameter.

\begin{tabular}{|c|c|c|c|c|}
\hline$E_{\beta}[\mathrm{keV}]$ & $E_{\gamma}[\mathrm{keV}]$ & $\epsilon[\mathrm{keV}]$ & $a_{2} / \beta_{0}$ & $\alpha_{L}\left[\mathrm{keV}^{-1}\right]$ \\
\hline 1769 & 1314 & 81.3 & 0.333 & $10^{-4}$ \\
\hline
\end{tabular}

Table 2: Parameters of the TRVM in the present calculations for ${ }^{126}$ Xe. $E_{\beta}$ and $E_{\gamma}$ are the vibration energies, $\epsilon$ is the inverse moment of inertia and $a_{2} / \beta_{0}$ the relative ratio of the static $\gamma$ and $\beta$ deformations. $\alpha_{L}$ is the Lipas parameter.

\begin{tabular}{|c|c|c|c|}
\hline$I_{i} \rightarrow I_{f}$ & $\mathrm{IBA}-\mathrm{CQF}$ & exp.13 & TRVM \\
\hline $2_{2}^{+} \rightarrow 0_{1}^{+}$ & 6.2 & $6.2 \pm 0.7$ & 4.5 \\
\hline $2_{1}^{+}$ & 100.0 & 100.0 & 100.0 \\
\hline $3_{1}^{+} \rightarrow 2_{2}^{+}$ & 100.0 & 100.0 & 100.0 \\
\hline $4_{1}^{+}$ & 29 & $22.0 \pm 3.0$ & 16 \\
\hline $2_{1}^{+}$ & 6.1 & $4.5 \pm 0.6$ & 2.6 \\
\hline $4_{2}^{+} \rightarrow 2_{2}^{+}$ & 100.0 & 100.0 & 100.0 \\
\hline $4_{1}^{+}$ & 58 & $54.0 \pm 10.0$ & 57 \\
\hline $2_{1}^{+}$ & 0.12 & $2.3 \pm 0.4$ & 2.75 \\
\hline $0_{2}^{+} \rightarrow 2_{2}^{+}$ & 100.0 & 100.0 & 100.0 \\
\hline $2_{1}^{+}$ & 2.5 & $3.3 \pm 0.2$ & 3.0 \\
\hline $2_{3}^{+} \rightarrow 0_{2}^{+}$ & 100.0 & 100.0 & 100.0 \\
\hline $2_{2}^{+}$ & 0.11 & $21.0 \pm 4.0$ & 0.04 \\
\hline $4_{1}^{+}$ & 1.3 & $2.7 \pm 0.5$ & 0.11 \\
\hline $2_{1}^{+}$ & 0.0 & $3.3 \pm 0.6$ & 0.06 \\
\hline & 0.24 & $0.017 \pm 0.003$ & 0.04 \\
\hline & 112 & & 18 \\
\hline $4_{3}^{+} \rightarrow 2_{2}^{+}$ & 0.02 & $2.9(5)$ & 670 \\
\hline & 108 & $97(17)$ & 214 \\
\hline & 100.0 & $100.0^{*}$ & 100.0 \\
\hline $4_{1}^{+}$ & 0.01 & $3.4(6)^{*}$ & 7.2 \\
\hline $2_{1}^{+}$ & 0.0 & $0.30(6)$ & 23.3 \\
\hline
\end{tabular}

Table 3: $\mathrm{B}(\mathrm{E} 2)$ branching ratios of the TRVM for ${ }^{130} \mathrm{Ba}$ in comparison with IBA and with experiment from Ref. [13]. 


\begin{tabular}{|c|c|c|c|}
\hline$I_{i} \rightarrow I_{f}$ & IBA $\mathrm{O}(6)_{\chi, \gamma}$ & $\exp \cdot[1]$ & TRVM \\
\hline $2_{2}^{+} \rightarrow 0_{1}^{+}$ & 1.51 & $1.5 \pm 0.4$ & 8.7 \\
\hline $2_{1}^{+}$ & 100.0 & 100.0 & 100.0 \\
\hline $3_{1}^{+} \rightarrow 2_{2}^{+}$ & 100.0 & 100.0 & 100.0 \\
\hline $4_{1}^{+}$ & 41 & $34.0_{-34}^{+10}$ & 24 \\
\hline $2_{1}^{+}$ & 1.9 & $2.0_{-1.7}^{+0.6}$ & 4.4 \\
\hline $4_{2}^{+} \rightarrow 2_{2}^{+}$ & 100.0 & 100.0 & 100.0 \\
\hline $4_{1}^{+}$ & 89 & $76.0 \pm 22.0$ & 66 \\
\hline $2_{1}^{+}$ & 2.1 & $0.4 \pm 0.1$ & 1.5 \\
\hline $0_{2}^{+} \rightarrow 2_{2}^{+}$ & 100.0 & 100.0 & 100.0 \\
\hline $2_{1}^{+}$ & 2.4 & $7.7 \pm 2.2$ & 1.1 \\
\hline $2_{3}^{+} \rightarrow 0_{2}^{+}$ & 100.0 & 100.0 & 100.0 \\
\hline $2_{2}^{+}$ & 2.7 & $2.2 \pm 1.0^{*}$ & 0.8 \\
\hline $4_{1}^{+}$ & 0.95 & $2.0 \pm 0.8$ & 0.04 \\
\hline $2_{1}^{+}$ & 0.8 & $0.14 \pm 0.06^{*}$ & 0.01 \\
\hline $0_{1}^{+}$ & 0.01 & $0.13 \pm 0.04$ & 0.01 \\
\hline $3_{1}^{+}$ & 86 & $67.0 \pm 22.0^{*}$ & 20 \\
\hline $5_{1}^{+} \rightarrow 6_{1}^{+}$ & 49 & $75 \pm 23$ & 43 \\
\hline $4_{2}^{+}$ & 52 & $76 \pm 21$ & 83 \\
\hline $3_{1}^{+}$ & 100.0 & 100.0 & 100.0 \\
\hline $4_{1}^{+}$ & 2.3 & $2.9 \pm 0.8$ & 0.8 \\
\hline $6_{2}^{+} \rightarrow 6_{1}^{+}$ & 43 & $34_{-25}^{+15}$ & 22 \\
\hline $4_{2}^{+}$ & 100.0 & 100.0 & 100.0 \\
\hline $4_{1}^{+}$ & 1.0 & $0.49 \pm 0.15$ & 1.3 \\
\hline $7_{1}^{+} \rightarrow 6_{2}^{+}$ & 20 & $40 \pm 26$ & 22 \\
\hline $5_{1}^{+}$ & 100.0 & 100.0 & 100.0 \\
\hline
\end{tabular}

Table 4: $\mathrm{B}(\mathrm{E} 2)$ branching ratios of the TRVM for ${ }^{126} \mathrm{Xe}$ in comparison with the IBA and with experiment from Ref.[1]. 